\title{
Preparation and Determination of the Absolute Configuration of Optically Active 9-Hydroxy-7-oxabicyclo[4.3.0]non-4-en-8-ones
}

\author{
David E. Hibbs, ${ }^{a}$ Michael B. Hursthouse, ${ }^{a}$ Lars J. S. Knutsen, ${ }^{\dagger, b}$ K. M. Abdul Malik, ${ }^{a}$ \\ Horacio F. Olivo, ${ }^{c}$ Stanley M. Roberts, ${ }^{c, *}$ David R. Varley ${ }^{c}$ and Hui Xiong ${ }^{c}$
}

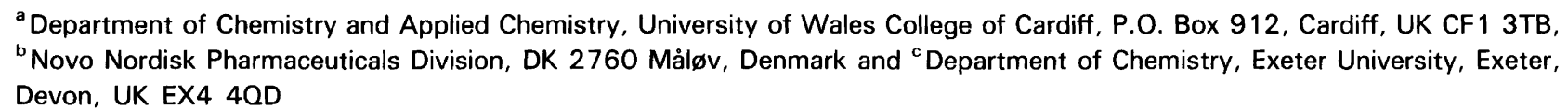

\begin{abstract}
Hibbs, D. E., Hursthouse, M. B., Knutsen, L. J. S., Abdul Malik, K. M., Olivo, H. F., Roberts, S. M., Varley, D. R. and Xiong, H., 1995. Preparation and Determination of the Absolute Configuration of Optically Active 9-Hydroxy-7oxabicyclo[4.3.0]non-4-en-8-ones. - Acta Chem. Scand. 49: 122-127 () Acta Chemica Scandinavica 1995

Both enantiomers of endo-9-hydroxy-7-oxabicyclo[4.3.0]non-4-en-8-one $(+)-(3)$ and (-)-(3) as well as exo-(1R,6R,9R)-9-hydroxy-7-oxabicyclo[4.3.0]non-4-en-8one $(+)-(4)$ have been obtained in states of high optical purity by kinetic resolutions involving enzyme-catalysed hydrolyses. Absolute configurations were assigned by chemical interconversions and by X-ray crystallography on Mosher's ester $(+)-(7)$.
\end{abstract}

The bicyclic lactones $\mathbf{1}$ and $\mathbf{2}$ are readily prepared, ${ }^{1}$ easily resolved $^{2}$ and have been shown to be very useful synthons. ${ }^{3-5}$ The homologous compounds 3 and 4 are also available $^{1}$ (following the procedure originally described by Lubineau et al. ${ }^{1}$ ), and recently we have investigated the resolution of these compounds prior to their use in synthetic schemes. ${ }^{6}$ Herein we report the results of these investigations.

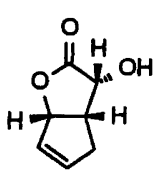

1

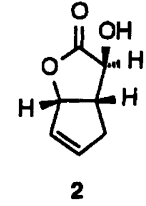

2

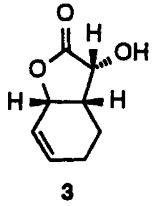

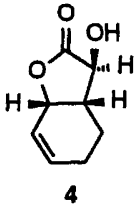

4

\section{Results and discussion}

The endo-hydroxy lactone $( \pm)-3$ was treated with Pseudomonas fluorescens lipase (Pfl) in vinyl acetate to afford the unchanged alcohol (+)-3 and the ester (-)-5 (Scheme 1). This ester was hydrolysed in aqueous phosphate buffer using $\mathrm{Pfl}$ as catalyst to yield the alcohol (-)-3.

Surprisingly the exo-hydroxy lactone $( \pm)-4$ is unaffected by Pfl in vinyl acetate. This racemic hydroxy lactone is best resolved using Candida cylindracea lipase

\footnotetext{
$\lceil$ To whom correspondence should be addressed.
}

$(\mathrm{Ccl})$ in vinyl acetate to give unchanged alcohol (-)-4 and ester (-)-6. However this biotransformation is extremely slow, taking 21 days to effect a $13 \%$ conversion.

The alcohols $(+)-3$ and $(-)-3$ were converted into the esters $(+)-7$ and $(+)-8$ by Mitsunobu reactions using $(R)-(+)$-Mosher acid. Acylation of the hydroxy lactone ( +)-4 [obtained by hydrolysis of the ester ( - )-6 using $\mathrm{Ccl}$ in $\mathrm{pH} 6.5$ buffer at room temp. over $24 \mathrm{~h}$ ] using $(S)$-Mosher acid chloride also gave the ester (+)-8.

Crystallographic study. The structure and absolute configuration of the ester (+)-7 was elucidated by X-ray crystallography, the latter by reference to the known chirality at $\mathrm{C}(1)$. The structure of a single molecule of 7 together with the atom numbering is shown in Fig. 1. The crystal data, molecular geometry parameters and the atom coordinates are given in Tables 1, 2 and 3, respectively.

The molecular dimensions are mostly as expected. The carbonyl angles $\mathrm{O}(2)=\mathrm{C}(10)-\mathrm{C}(1) / \mathrm{O}(3)$ are nearly equal at ca. $125^{\circ}$ but the $\mathrm{O}(4)=\mathrm{C}(12)-\mathrm{C}(11) / \mathrm{O}(5)$ angles are quite asymmetric with values $128.2(2)$ and $123.3(2)^{\circ}$. The widening of the $\mathrm{O}(4)-\mathrm{C}(12)-\mathrm{C}(11)$ angle may be attributed to the short intramolecular $\mathrm{O}(4) \cdots \mathrm{O}(3)$ contact of 2.848(3) A. Intramolecular short contacts involving C(9) [with $\mathrm{C}(2)$ and $\mathrm{C}(3)$ ] and $\mathrm{O}(2)$ [with $\mathrm{H}(11)$ ] may appear to be responsible for widening of the tetrahedral angles at $\mathrm{O}(1)\left[118.4(2)^{\circ}\right]$ and at $\mathrm{O}(3)\left[116.9(2)^{\circ}\right]$. There are no unusually short intermolecular contacts. 


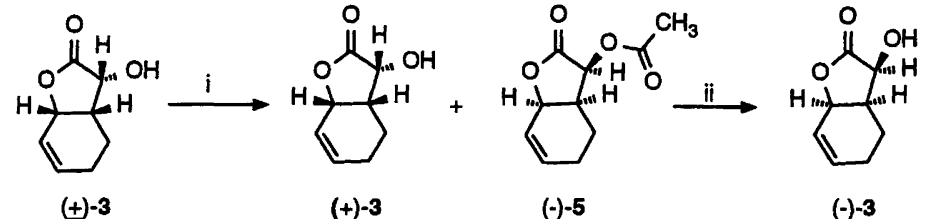

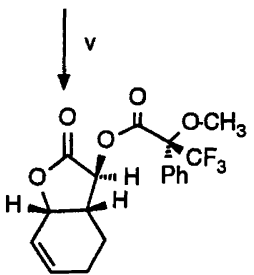

$(+)-7$

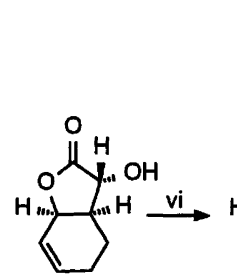

$-(+)-4$
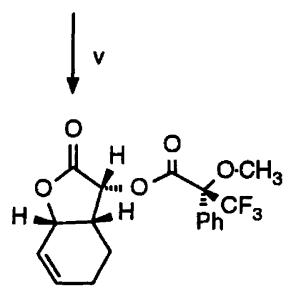

$(+)-8$

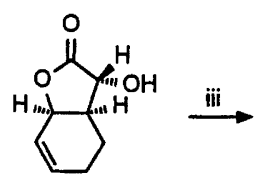

$(+-4$

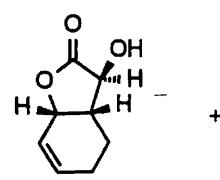

$(-)-4$

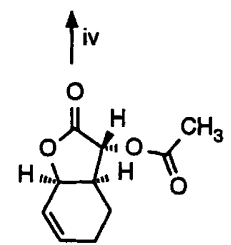

$(-)-6$

Scheme 1. Reagents and conditions: (i) Pfl, vinyl acetate, $70 \mathrm{~h}$; (ii) Pfl, phosphate buffer, $20 \mathrm{~h}$; (iii) Ccl, vinyl acetate, 21 days, $30^{\circ} \mathrm{C}$; (iv) $\mathrm{Ccl}$, phosphate buffer, $24 \mathrm{~h}$; (v) diethyl azodicarboxylate, $\mathrm{Ph}_{3} \mathrm{P}$, (R)-Mosher acid, tetrahydrofuran; (vi) (S)-Mosher acid chloride, $\mathrm{CH}_{2} \mathrm{Cl}_{2}$, pyridine.

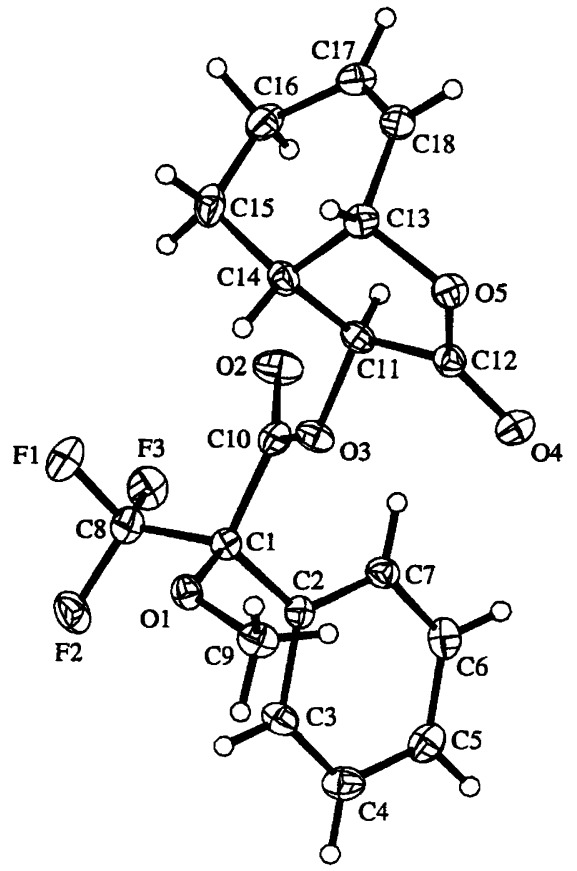

Fig. 1. Molecular structure and absolute configuration of $(+)-(7)$ showing the numbering scheme of the non-hydrogen atoms. The hydrogen atoms are represented by small circles.

Conclusions. Now that the optically pure compounds $(+)-3$ and $(-)-3$ are readily available, these bicyclic lactones can be considered as potential intermediates for the preparation of novel homochiral compounds.

\section{Experimental}

IR spectra were recorded on a Nicolet Magna-IR 550 spectrometer. Optical rotations were measured on an Optical Instrument A-1000 polarimeter. ${ }^{1} \mathrm{H}$ and ${ }^{13} \mathrm{C}$ NMR spectra were recorded on a Bruker AC-300 spectrometer. Chemical shifts are given in ppm and coupling constants in $\mathrm{Hz}$. Mass spectra were recorded at the SERC Mass Spectrometry Centre, Swansea on a VG ZAB-F spectrometer (high resolution), or a VG 12-253 spectrometer (low resolution). Measurements of enantiomeric excesses were measured by NMR spectroscopy using tris[3(heptafluoropropylhydroxymethylene)-(+)-camphorato]europium(III) as the chiral shift reagent (error $\pm 2 \%$ ). Enzymes were obtained from Biocatalysts, Sketty, Swansea, UK.

Enzymatic acetylation of endo-9-hydroxy-7-oxabicyclo[4.3.0]non-4-en-8-one ( \pm )-3. Pseudomonas fluorescens lipase $(1.00 \mathrm{~g})$ was added to a solution of $( \pm)$-endo-9-hydroxy-7-oxabicyclo[4.3.0]non-4-en-8-one $3(1.54 \mathrm{~g}$, $10.0 \mathrm{mmol})$ in vinyl acetate $(50 \mathrm{ml})$. The mixture was shaken for $70 \mathrm{~h}$ in an orbital incubator at $30^{\circ} \mathrm{C}$ and then the enzyme was removed by filtration. The filtrate was concentrated under reduced pressure and the residue was chromatographed over silica gel (ethyl acetate-hexane 1:1). The first fractions gave (-)-endo- $(1 R, 6 R, 9 S)-9$-acetoxy-7-oxabicyclo[4.3.0]non-4-en-8-one ( - )-5 $(0.911 \mathrm{~g}$, $4.64 \mathrm{mmol}, 46.3 \%$ ) as white crystals. $R_{\mathrm{f}} 0.51$ (ethyl acetate-hexane $1: 1$ ), m.p. $74.2^{\circ} \mathrm{C}$ (ethyl acetate); $[\alpha]_{\mathrm{D}}^{25}$ -22.3 (c 1.0 in $\mathrm{CHCl}_{3}$ ), 100\% ee. (Found: 197.08177, 
Table 1. Crystal data and details of data collection and structure refinement for $\mathrm{C}_{18} \mathrm{H}_{17} \mathrm{~F}_{3} \mathrm{O}_{5} 7$

\begin{tabular}{|c|c|}
\hline $\begin{array}{l}\text { Empirical formula } \\
\text { Formula weight } \\
\text { Temperature } \\
\text { Radiation } \\
\text { Wavelength } \\
\text { Crystal system } \\
\text { Space group } \\
\text { Unit cell dimensions } \\
\\
\text { Volume } \\
Z \\
\text { Density (calculated) } \\
\text { Absorption coefficient (Mo K } \alpha \text { ) } \\
F(000) \\
\text { Crystal size } \\
\theta \text { range for data collection } \\
\text { Index ranges } \\
\text { Reflections collected } \\
\text { Absorption correction factors } \\
\text { Independent reflections } \\
\text { Refinement method } \\
\text { Data/parameters } \\
\text { Goodness-of-fit on } F^{2} \\
\left.\text { Final } R^{a} \text { indices [I>2 } \sigma(/)\right] \\
R^{*} \text { indices (all data) } \\
\text { Largest diff. peak and hole }\end{array}$ & $\begin{array}{l}\mathrm{C}_{18} \mathrm{H}_{17} \mathrm{~F}_{3} \mathrm{O}_{5} \\
370.32 \\
140(2) \mathrm{K} \\
\mathrm{Mo}-\mathrm{K} \alpha \\
0.71069 \AA \\
\text { Orthorhombic } \\
P 2{ }_{1},_{1}, \\
a=6.8310(10) \AA \\
b=11.481(2) \AA \\
c=21.788(6) \AA \\
1708.8(6) \AA^{3} \\
4 \\
1.439 \mathrm{Mg} \mathrm{m}^{-3} \\
0.125 \mathrm{~mm}^{-1} \\
768 \\
0.30 \times 0.15 \times 0.10 \mathrm{~mm} \\
1.87-24.93^{\circ} \\
-7 \leqslant h \leqslant 5,-12 \leqslant k \leqslant 12,-23 \leqslant I \leqslant 22 \\
6543 \\
0.897-1.015 \\
2577\left[R_{\text {int }}=0.0539\right] \\
\text { Full-matrix least-squares on } F^{2} \\
2577 / 236 \\
0.927 \\
R_{1}=0.0323, w R_{2}=0.0673 \\
R_{1}=0.0384, w R_{2}=0.0695 \\
0.160 \text { and }-0.177 \text { e } \dot{A}^{-3}\end{array}$ \\
\hline
\end{tabular}

${ }^{a} R_{1}=\Sigma(\Delta F) / \Sigma\left(F_{0}\right) ; \quad W R_{2}=\left(\zeta\left\{W\left[\Delta\left(F^{2}\right)^{2}\right]\right\} / \Sigma\left\{w\left(F_{0}^{2}\right)^{2}\right\}\right)^{1 / 2} ; \quad W=1 /\left[\sigma^{2}\left(F_{0}^{2}\right)+(0.0268 P)^{2}\right], \quad$ where $\quad P=\left[\operatorname{Max}\left(F_{0}^{2}\right)+2\left(F_{0}^{2}\right)+\right.$ $\left.2\left(F_{0}^{2}\right)\right] / 3$.

$\mathrm{C}_{10} \mathrm{H}_{12} \mathrm{O}_{4}+\mathrm{H}$ requires 197.08137). IR ( $\mathrm{KBr}$ disc): 3046 , $2980,2955,2930,1772,1751,1652,1382,1234$, $1100 \mathrm{~cm}^{-1} ;{ }^{1} \mathrm{H}$ NMR $\left(300 \mathrm{MHz} ; \mathrm{CDCl}_{3}\right): \delta 6.25(1 \mathrm{H}$, $\mathrm{m}, 4-\mathrm{H}), 5.92(1 \mathrm{H}, \mathrm{m}, 5-\mathrm{H}), 5.65(1 \mathrm{H}, \mathrm{d}, J$ 7.5, 9-H), $4.72(1 \mathrm{H}, \mathrm{t}, J 4.5,6-\mathrm{H}), 2.84(1 \mathrm{H}, \mathrm{m}, 1-\mathrm{H}), 2.2(4 \mathrm{H}$, $\mathrm{m}, 3-\mathrm{H}$ and $\left.\mathrm{CH}_{3}\right), 2.0(1 \mathrm{H}, \mathrm{m}, 2-\mathrm{H}), 1.64\left(1 \mathrm{H}, \mathrm{m}, 3^{\prime}-\mathrm{H}\right)$ and $1.29\left(1 \mathrm{H}, \mathrm{m}, 2^{\prime}-\mathrm{H}\right) .{ }^{13} \mathrm{C} \mathrm{NMR}\left(75.5 \mathrm{MHz} ; \mathrm{CDCl}_{3}\right)$ : $\delta 171.70(\mathrm{CO}), 169.83(\mathrm{CO}), 136.08(\mathrm{CH}, \mathrm{C}-4), 121.62$ (CH, C-5), 71.82 (CH, C-6), 71.35 (CH, C-9), $37.61(\mathrm{CH}$, $\mathrm{C}-1), 22.96\left(\mathrm{CH}_{2}, \mathrm{C}-3\right), 20.40\left(\mathrm{CH}_{3}\right)$ and $17.76\left(\mathrm{CH}_{2}\right.$, $\mathrm{C}-2)$; MS (CI): $m / z 197(M+\mathrm{H}, 1.2 \%), 196\left(M^{+}, 0.1\right)$, $154\left(M-\mathrm{C}_{2} \mathrm{H}_{3} \mathrm{O}, 0.4\right), 137\left(M-\mathrm{C}_{2} \mathrm{H}_{3} \mathrm{O}_{2}, 7.0\right), 110$ (46.8), $109(10.2), 95(24.2), 93(31.6)$ and $92(100.0)$. Later fractions contained $(+)$-endo- $(1 S, 6 S, 9 R)-9$-hydroxy-7-oxabicyclo[4.3.0]non-4-en-8-one (+)-3 $(0.560 \mathrm{~g}, 3.63 \mathrm{mmol}$, $36.2 \%,[\alpha]_{\mathrm{D}}^{25}+143.9\left(\mathrm{c} 1.0\right.$ in $\left.\mathrm{CHCl}_{3}\right), 100 \%$ ee $) . R_{\mathrm{f}} 0.26$ (ethyl acetate-hexane $1: 1$ ); m.p. $131.0^{\circ} \mathrm{C}$ (ether). (Found: $M^{+} 172.0974, \mathrm{C}_{8} \mathrm{H}_{10} \mathrm{O}_{3}+\mathrm{NH}_{4}$ requires $M$ 172.0973). IR (KBr): 3448, 3044, 2965, 2942, 2916, 1761, 1201, 1110, 952 and $908 \mathrm{~cm}^{-1} ;{ }^{1} \mathrm{H}$ NMR $(300 \mathrm{MHz}): \delta 6.24(1 \mathrm{H}$, $\mathrm{m}, 4-\mathrm{H}), 5.93(1 \mathrm{H}, \mathrm{m}, 5-\mathrm{H}), 4.69(2 \mathrm{H}, \mathrm{m}, 6-\mathrm{H}$ and $9-\mathrm{H})$, $3.12(1 \mathrm{H}$, br s, OH), $2.7(1 \mathrm{H}, \mathrm{m}, 1-\mathrm{H}), 2.2(1 \mathrm{H}, \mathrm{m}$, 3-H), 2.07-1.98 (1 H, m, 2-H), 1.97-1.88 (1 H, m, 3'-H) and $1.28-1.12\left(1 \mathrm{H}, \mathrm{m}, 2{ }^{\prime}-\mathrm{H}\right) ;{ }^{13} \mathrm{C}$ NMR $(75.5 \mathrm{MHz}): \delta$ 177.23 (CO), 136.55 (CH, C-4), 121.64 (CH, C-5), 71.80 (CH, C-6), 71.50 (CH, C-9), 39.11 (CH, C-1), 23.17 $\left(\mathrm{CH}_{2}, \mathrm{C}-3\right), 17.29\left(\mathrm{CH}_{2}, \mathrm{C}-2\right) ; \mathrm{MS}(\mathrm{CI}): \mathrm{m} / z \quad 172$ $\left(M+\mathrm{NH}_{4}, 100 \%\right)$.
Enzymatic acetylation of exo-9-hydroxy-7-oxabicyclo[4.3.0]non-4-en-8-one ( \pm )-4. Candida cylindracea lipase $(0.17 \mathrm{~g})$ was added to a solution of $( \pm)$-exo-9-hydroxy7-oxabicyclo[4.3.0]non-4-en-8-one $4(295 \mathrm{mg}, \quad 1.68$ $\mathrm{mmol}$ ) in vinyl acetate $(25 \mathrm{ml})$. The mixture was shaken for 21 days in an orbital incubator at $30^{\circ} \mathrm{C}$ and then the enzyme was removed by filtration. The filtrate was concentrated under reduced pressure and the residue was chromatographed over silica gel (ethyl acetate-hexane $1: 1)$. The first fractions gave (-)-exo-(1R,6R,9R)-9-acetoxy-7-oxabicyclo[4.3.0]non-4-en-8-one ( - )-6 (43.3 mg, $0.22 \mathrm{mmol}, 13.1 \%$ ) as an oil. $R_{\mathrm{f}} 0.52$ (ethyl acetate-hexane $1: 1) .[\alpha]_{\mathrm{D}}^{25}-44.1$ (c 1.0 in $\mathrm{CHCl}_{3}$ ), $100 \%$ ee. (Found: $M^{+}$197.08106, $\mathrm{C}_{10} \mathrm{H}_{13} \mathrm{O}_{4}+\mathrm{H}$ requires $M$ 197.08137). IR (neat): 3030, 2939, 1790, 1750, 1235, 1082, 993 and $965 \mathrm{~cm}^{-1}$; ${ }^{1} \mathrm{H}$ NMR (300 MHz): $\delta 6.03(1 \mathrm{H}, \mathrm{dt}, 4-\mathrm{H})$, $5.75(1 \mathrm{H}, \mathrm{m}, 5-\mathrm{H}), 5.41(1 \mathrm{H}, \mathrm{d}, J 9,9-\mathrm{H}), 5.0(1 \mathrm{H}, \mathrm{m}$, 6-H), 2.75 (1 H, m, 1-H), 2.38-2.22 (1 H, m, 3-H), 2.14 $\left(4 \mathrm{H}, \mathrm{m}, 3^{\prime}-\mathrm{H}\right.$ and $\left.\mathrm{CH}_{3}\right)$ and $1.80(2 \mathrm{H}, \mathrm{m}, 2-\mathrm{H}) .{ }^{13} \mathrm{C}$ NMR (75.5 MHz): $\delta 172.33$ (CO), $169.94(\mathrm{CO}), 133.12$ (CH, C-4), 123.78 (CH, C-5), 73.75 (CH, C-6), 69.05 (CH, C-9), $39.27(\mathrm{CH}, \mathrm{C}-1), 20.57\left(\mathrm{CH}_{3}\right), 20.36\left(\mathrm{CH}_{2}\right.$, C-3), $20.25\left(\mathrm{CH}_{2}, \mathrm{C}-2\right)$; MS (CI): $\mathrm{m} / z \quad 197(M+\mathrm{H}$, $100 \%), 136(18), 118(29), 110(30)$ and $92(88)$. Later fractions contained exo-(1S,6S,9S)-9-hydroxy-7-oxabicyclo[4.3.0]non-4-en-8-one (- )-4 $(0.217 \mathrm{~g}, 1.40 \mathrm{mmol}$, $83.7 \%$ ). $R_{\mathrm{f}} 0.33$ (ethyl acetate-hexane $1: 1$ ); IR (neat): $3413,3039,2932,1771,1649,1441,1124,1088 \mathrm{~cm}^{-1} ;{ }^{1} \mathbf{H}$ 
Table 2. Bond lengths $(\dot{A})$ and angles $\left({ }^{\circ}\right)$ for $\mathrm{C}_{18} \mathrm{H}_{17} \mathrm{~F}_{3} \mathrm{O}_{5} 7$.

\begin{tabular}{|c|c|c|c|}
\hline $\begin{array}{l}C(1)-O(1) \\
C(1)-C(8) \\
C(2)-C(7) \\
C(3)-C(4) \\
C(5)-C(6) \\
C(8)-F(1) \\
C(8)-F(3) \\
C(10)-O(2) \\
C(11)-O(3) \\
C(11)-C(12) \\
C(12)-O(5) \\
C(13)-C(18) \\
C(14)-C(15) \\
C(16)-C(17)\end{array}$ & $\begin{array}{l}1.419(3) \\
1.540(3) \\
1.386(3) \\
1.376(3) \\
1.368(3) \\
1.339(3) \\
1.344(3) \\
1.200(3) \\
1.450(2) \\
1.525(3) \\
1.346(3) \\
1.484(3) \\
1.522(3) \\
1.499(3)\end{array}$ & $\begin{array}{l}C(1)-C(2) \\
C(1)-C(10) \\
C(2)-C(3) \\
C(4)-C(5) \\
C(6)-C(7) \\
C(8)-F(2) \\
C(9)-O(1) \\
C(10)-O(3) \\
C(11)-C(14) \\
C(12)-O(4) \\
C(13)-O(5) \\
C(13)-C(14) \\
C(15)-C(16) \\
C(17)-C(18)\end{array}$ & $\begin{array}{l}1.523(3) \\
1.541(3) \\
1.400(3) \\
1.394(3) \\
1.389(3) \\
1.339(3) \\
1.446(3) \\
1.341(3) \\
1.509(3) \\
1.202(3) \\
1.476(3) \\
1.537(3) \\
1.514(3) \\
1.315(3)\end{array}$ \\
\hline $\begin{array}{l}O(1)-C(1)-C(2) \\
C(2)-C(1)-C(8) \\
C(2)-C(1)-C(10) \\
C(7)-C(2)-C(3) \\
C(3)-C(2)-C(1) \\
C(3)-C(4)-C(5) \\
C(5)-C(6)-C(7) \\
F(1)-C(8)-F(2) \\
F(2)-C(8)-F(3) \\
F(2)-C(8)-C(1) \\
O(2)-C(10)-O(3) \\
O(3)-C(10)-C(1) \\
O(3)-C(11)-C(12) \\
O(4)-C(12)-O(5) \\
O(5)-C(12)-C(11) \\
O(5)-C(13)-C(14) \\
C(11)-C(14)-C(15) \\
C(15)-C(14)-C(13) \\
C(17)-C(16)-C(15) \\
C(17)-C(18)-C(13) \\
C(10)-O(3)-C(11)\end{array}$ & $\begin{array}{l}113.7(2) \\
109.9(2) \\
111.9(2) \\
118.7(2) \\
118.5(2) \\
120.1(2) \\
120.9(2) \\
106.8(2) \\
106.5(2) \\
111.7(2) \\
124.6(2) \\
110.7(2) \\
108.0(2) \\
123.3(2) \\
108.5(2) \\
104.7(2) \\
116.5(2) \\
112.2(2) \\
112.0(2) \\
124.0(2) \\
116.9(2)\end{array}$ & $\begin{array}{l}O(1)-C(1)-C(8) \\
O(1)-C(1)-C(10) \\
C(8)-C(1)-C(10) \\
C(7)-C(2)-C(1) \\
C(4)-C(3)-C(2) \\
C(6)-C(5)-C(4) \\
C(2)-C(7)-C(6) \\
F(1)-C(8)-F(3) \\
F(1)-C(8)-C(1) \\
F(3)-C(8)-C(1) \\
O(2)-C(10)-C(11) \\
O(3)-C(11)-C(14) \\
C(14)-C(11)-C(12) \\
O(4)-C(12)-C(11) \\
O(5)-C(13)-C(18) \\
C(18)-C(13)-C(14) \\
C(11)-C(14)-C(13) \\
C(16)-C(15)-C(14) \\
C(18)-C(17)-C(16) \\
C(1)-O(1)-C(9) \\
C(12)-O(5)-C(13)\end{array}$ & $\begin{array}{l}101.4(2) \\
112.2(2) \\
107.0(2) \\
122.7(2) \\
120.6(2) \\
119.5(2) \\
120.2(2) \\
107.3(2) \\
112.1(2) \\
112.1(2) \\
124.7(2) \\
112.8(2) \\
104.3(2) \\
128.2(2) \\
109.4(2) \\
113.1(2) \\
100.4(2) \\
113.0(2) \\
124.2(2) \\
118.4(2) \\
110.2(2)\end{array}$ \\
\hline
\end{tabular}

NMR (300 MHz): $\delta 6.0(1 \mathrm{H}, \mathrm{m}, 4-\mathrm{H}), 5.77(1 \mathrm{H}, \mathrm{m}$, 5-H), $5.0(1 \mathrm{H}, \mathrm{m}, 6-\mathrm{H}), 4.3(1 \mathrm{H}, \mathrm{dd}, J 10,4,9-\mathrm{H}), 3.54$ (1 H, m, OH), $2.7(1 \mathrm{H}, \mathrm{m}, 1-\mathrm{H}), 2.28-1.78(4 \mathrm{H}, \mathrm{m}, 2-\mathrm{H}$ and $3-\mathrm{H}) ;{ }^{13} \mathrm{C}$ NMR (75.5 MHz) 177.76 (CO), 132.75 (CH, C-4), 124.28 (CH, C-5), 74.22 (CH, C-6), 68.50 (CH, C-9), 40.67 (CH, C-1), $20.16\left(\mathrm{CH}_{2}, \mathrm{C}-3\right)$ and 20.13 $\left(\mathrm{CH}_{2}, \mathrm{C}-2\right)$; MS (EI): $m / z 154(M, 0.9 \%), 153(M-\mathrm{H}$, 3.8), $110\left(M-\mathrm{CO}_{2}, 45\right), 92(41)$ and $79(100)$.

Enzymatic hydrolysis of (-)-endo-(1 S,6R,9S)-9-acetoxy-7oxabicyclo[4.3.0]non-4-en-8-one (-)-5 and (-)-exo-9-acetoxy-7-oxabicyclo[4.3.0]non-4-en-8-one (-)-6. Pseudomonas fluorescens lipase $(33 \mathrm{mg})$ was added to a solution of ester (- )-endo-(1R,6R,9S)-9-acetoxy-7-oxabicyclo[4.3.0]non-4-en-8-one (-)-5 (0.200 g, $1.01 \mathrm{mmol}, 100 \%$ ee) in a mixture of THF $(2.5 \mathrm{ml})$ and aqueous phosphate buffer $\left(0.1 \mathrm{~mol} \mathrm{l}^{-1}, 5.0 \mathrm{ml}, \mathrm{pH} 7.0\right)$. The mixture was stirred for $20 \mathrm{~h}$ at room temperature. During the reaction aqueous sodium hydroxide $\left(1 \mathrm{~mol} 1^{-1}, 0.6 \mathrm{ml}\right)$ was added to the mixture at 30-minute intervals to maintain the $\mathrm{pH}$ between 6.5 and 7.0. The lipase was removed by filtration and the filtrate was saturated with sodium chloride and extracted with ethyl acetate $(10 \times 10 \mathrm{ml})$. The extract was concentrated under reduced pressure and the residue was chromatographed over silica gel (ethyl acetate-hexane $3: 2)$ to give alcohol (-)-endo-(1R,6R,9S)-9-hydroxy-7oxabicyclo[4.3.0]non-4-en-8-one (-)-3 \{115 mg, $0.746 \mathrm{mmol}, 73.2 \%,[\alpha]_{\mathrm{D}}^{27}-143.4$ (c 1.0 in $\mathrm{CHCl}_{3}$ ); $100 \%$ ee . Treatment of the acetate $(-)-6(30 \mathrm{mg})$ with Candida cylindracea lipase in $\mathrm{pH} 6.5$ buffer at room temperature for $24 \mathrm{~h}$ with work-up as above gave the hydroxy lactone $(+)-4[\alpha]_{\mathrm{D}}^{23}+5.7\left(c 0.5\right.$ in $\left.\mathrm{CHCl}_{3}\right)$ in $48 \%$ yield and $100 \%$ ee.

Preparation of Mosher ester $(+)-\mathbf{8}$ by acylation. Hydroxy lactone $(+)-4(6.6 \mathrm{mg})$ in dichloromethane $(2 \mathrm{ml})$ was reacted with $(+)-(S)-\alpha$-methoxy- $\alpha$-trifluoromethylphenylacetyl chloride $(67.2 \mathrm{mg}, 0.28 \mathrm{mmol})$ and dry pyridine $(175 \mathrm{mg}, 2.2 \mathrm{mmol})$ at room temperature for $12 \mathrm{~h}$ under nitrogen. The reaction mixture was diluted with dichloromethane $(10 \mathrm{ml})$ and washed with an aqueous copper sulfate solution $(2 \times 3 \mathrm{ml})$, sodium hydrogen carbonate $(2 \times 3 \mathrm{ml})$ and brine $(2 \times 3 \mathrm{ml})$. The combined organic layers were dried over anhydrous sodium sulfate and the solvent evaporated off under reduced pressure to provide ( + )-(1R,6R,9R)-9-[(R)- $\alpha$-methoxy- $\alpha$-trifluoromethyl- 
Table 3. Atomic coordinates $\left(\times 10^{4}\right)$ and equivalent isotropic displacement parameters $\left(\AA^{2} \times 10^{3}\right)$ for $\mathrm{C}_{18} \mathrm{H}_{17} \mathrm{~F}_{3} \mathrm{O}_{5} 7$.

\begin{tabular}{lrlll}
\hline & \multicolumn{1}{l}{$x$} & $y$ & $z$ & $U_{\text {eq }}{ }^{a}$ \\
\hline $\mathrm{C}(1)$ & $1485(3)$ & $4373(2)$ & $6647(1)$ & $23(1)$ \\
$\mathrm{C}(2)$ & $313(3)$ & $3609(2)$ & $6209(1)$ & $23(1)$ \\
$\mathrm{C}(3)$ & $256(3)$ & $2408(2)$ & $6316(1)$ & $30(1)$ \\
$\mathrm{C}(4)$ & $-808(3)$ & $1685(2)$ & $5939(1)$ & $35(1)$ \\
$\mathrm{C}(5)$ & $-1861(3)$ & $2147(2)$ & $5448(1)$ & $34(1)$ \\
$\mathrm{C}(6)$ & $-1839(3)$ & $3323(2)$ & $5348(1)$ & $32(1)$ \\
$\mathrm{C}(7)$ & $-750(3)$ & $4059(2)$ & $5722(1)$ & $26(1)$ \\
$\mathrm{C}(8)$ & $309(3)$ & $4581(2)$ & $7240(1)$ & $32(1)$ \\
$\mathrm{C}(9)$ & $4533(3)$ & $3293(2)$ & $6450(1)$ & $32(1)$ \\
$\mathrm{C}(10)$ & $1926(3)$ & $5579(2)$ & $6368(1)$ & $26(1)$ \\
$\mathrm{C}(11)$ & $4410(3)$ & $6715(2)$ & $5891(1)$ & $24(1)$ \\
$\mathrm{C}(12)$ & $5732(3)$ & $6467(2)$ & $5343(1)$ & $26(1)$ \\
$\mathrm{C}(13)$ & $7036(3)$ & $8023(2)$ & $5882(1)$ & $28(1)$ \\
$\mathrm{C}(14)$ & $5705(3)$ & $7369(2)$ & $6334(1)$ & $28(1)$ \\
$\mathrm{C}(15)$ & $4681(4)$ & $8189(2)$ & $6779(1)$ & $36(1)$ \\
$\mathrm{C}(16)$ & $3549(4)$ & $9147(2)$ & $6459(1)$ & $36(1)$ \\
$\mathrm{C}(17)$ & $4657(3)$ & $9644(2)$ & $5927(1)$ & $33(1)$ \\
$\mathrm{C}(18)$ & $6202(3)$ & $9152(2)$ & $5677(1)$ & $31(1)$ \\
$\mathrm{O}(1)$ & $3193(2)$ & $3826(1)$ & $6880(1)$ & $27(1)$ \\
$\mathrm{O}(2)$ & $793(2)$ & $6378(1)$ & $6355(1)$ & $39(1)$ \\
$\mathrm{O}(3)$ & $3741(2)$ & $5609(1)$ & $6134(1)$ & $28(1)$ \\
$\mathrm{O}(4)$ & $5555(2)$ & $5723(1)$ & $4959(1)$ & $34(1)$ \\
$\mathrm{O}(5)$ & $7220(2)$ & $7235(1)$ & $5350(1)$ & $30(1)$ \\
$\mathrm{F}(1)$ & $1201(2)$ & $5336(1)$ & $7615(1)$ & $45(1)$ \\
$\mathrm{F}(2)$ & $59(2)$ & $3595(1)$ & $7558(1)$ & $41(1)$ \\
$\mathrm{F}(3)$ & $-1490(2)$ & $5002(1)$ & $7125(1)$ & $39(1)$ \\
\hline
\end{tabular}

${ }^{a} U_{\mathrm{eq}}$ is defined as one third of the trace of the orthogonalized $U_{i j}$ tensor.

phenylacetoxy]-7-oxabicyclo[4-3.0]non-4-en-8-one (+)-8. $R_{\mathrm{f}} 0.34$ (ethyl acetate-hexane 1:4); m.p. $90-91^{\circ} \mathrm{C}$ (toluene-light petroleum); $[\alpha]_{\mathrm{D}}^{27}+47.1$ (c 1.0 in $\mathrm{CHCl}_{3}$ ). (Found: 370.10221, $\mathrm{C}_{18} \mathrm{H}_{17} \mathrm{~F}_{3} \mathrm{O}_{5}$ requires 370.10280); IR (neat): 2946, 1797, 1766, 1459, 1336, 1257 and $1174 \mathrm{~cm}^{-1} ;{ }^{19} \mathrm{~F}$ NMR: $\delta$ 90.0831; ${ }^{1} \mathrm{H}$ NMR $\left(\mathrm{CDCl}_{3}\right): \delta$ $7.60(2 \mathrm{H}, \mathrm{m}, \mathrm{Ph}), 7.42$ (3 H, m, Ph), 6.08 (1 H, m, J 2.6, 4.6, 4-H), $5.80(1 \mathrm{H}, \mathrm{m}, J 10.3,2.7,2.4,1.6,0.6,5-\mathrm{H})$, 5.72 (1 H, J 9.9, 9-H), 5.01 (1 H, m, $J$ 7.5, 2.4, 2.2, 6-H), $3.63\left(3 \mathrm{H}, \mathrm{OCH}_{3}\right), 2.80(1 \mathrm{H}, \mathrm{m}, J 9.9,7.5,4.6,4.0,0.6$, 1-H), 2.42-2.28 (1 H, m, 3-H), 2.23-2.11 (1 H, m, 3'-H) and $1.91-1.70(2 \mathrm{H}, \mathrm{m}, 2-\mathrm{H}) ;{ }^{13} \mathrm{C}$ NMR (75.5 MHz): $\delta$ 171.07 (CO, C-8), 166.16 (CO), 132.98 (CH, C-4), 131.71 $(\mathrm{C}, \mathrm{Ph}), 129.88(\mathrm{CH}, \mathrm{Ph}), 128.56(\mathrm{CH}, \mathrm{Ph}), 127.31(\mathrm{CH}$, $\mathrm{Ph}), 122.91$ (CH, C-5), $123.12\left(\mathrm{C}, \mathrm{q}, J_{\mathrm{CF}} 289, \mathrm{CF}_{3}\right), 84.86$ $\left(\mathrm{C}, J_{\mathrm{CCF}} 28, \mathrm{CCF}_{3}\right), 73.90(\mathrm{CH}, \mathrm{C}-6), 69.98(\mathrm{CH}, \mathrm{C}-9)$, $55.71\left(\mathrm{CH}, \mathrm{OCH}_{3}\right), 38.81(\mathrm{CH}, \mathrm{C}-1), 20.08\left(\mathrm{CH}_{2}, \mathrm{C}-3\right)$ and $20.05\left(\mathrm{CH}_{2}, \mathrm{C}-2\right)$; $\mathrm{MS}(\mathrm{EI}): \mathrm{m} / z 370\left(M^{+}, 0.2 \%\right)$, $301\left(M-\mathrm{CF}_{3}, 0.5\right), 190(9.8), 189\left[\mathrm{C}_{6} \mathrm{H}_{5} \mathrm{C}\left(\mathrm{CF}_{3}\right)\left(\mathrm{OCH}_{3}\right)\right.$, 100.0], 152 (4.7), 137 (1.5), 127 (2.2) and 105 (12.2).

General procedure for the Mitsunobu reaction using (+)(R)-Mosher acid. Diethyl azodicarboxylate $(3.45 \mathrm{mmol})$ in dry tetrahydrofuran (THF) $(10 \mathrm{ml})$ was added dropwise to a mixture of hydroxy lactone $(2.0 \mathrm{mmol})$, triphenylphosphine $(3.44 \mathrm{mmol})$ and $(+)-(R)$-Mosher acid
$(6.09 \mathrm{mmol})$ in dry THF $(20 \mathrm{ml})$. The mixture was stirred overnight at room temperature. Solvents were evaporated off under reduced pressure and the residue was purified by column chromatography (ethyl acetate-light petroleum 1:4). Reaction of hydroxy lactone $(+)-3$ under these conditions provided the ester (+)-7. M.p. $95-96^{\circ} \mathrm{C}$ (toluene-light petroleum), $\boldsymbol{R}_{\mathrm{f}} 0.28$ (1:4 ethyl acetate-hexane); $[\alpha]_{\mathrm{D}}^{27}+5.1\left(c 1.0\right.$ in $\left.\mathrm{CHCl}_{3}\right)$; IR (neat): 2946, 1793, 1758, 1459, 1331, 1255 and $1174 \mathrm{~cm}^{-1} ;{ }^{19} \mathrm{~F}$ NMR: $\delta 89.4861$; ${ }^{1} \mathrm{H}$ NMR (300 MHz; $\left.\mathrm{CDCl}_{3}\right): \delta 7.61(2 \mathrm{H}, \mathrm{m}, \mathrm{Ph}), 7.43$ ( $3 \mathrm{H}, \mathrm{m}, \mathrm{Ph}), 6.08(1 \mathrm{H}, \mathrm{m}, 4-\mathrm{H}), 5.81(1 \mathrm{H}, \mathrm{m}, 5-\mathrm{H}), 5.60$ (1 H, d, 9-H), $5.06(1 \mathrm{H}, \mathrm{m}, 6-\mathrm{H}), 3.54\left(3 \mathrm{H}, \mathrm{OCH}_{3}\right), 2.95$ (1 H, m, 1-H), 2.41-2.27 (1 H, m, 3-H), 2.23-2.11 (1 H, $\left.\mathrm{m}, 3^{\prime}-\mathrm{H}\right)$ and $1.97-1.77(2 \mathrm{H}, \mathrm{m}, 2-\mathrm{H}) ;{ }^{13} \mathrm{C}$ NMR: $\delta$ 170.67 (CO, C-8), 165.98 (CO), 132.95 (CH, C-4), 131.20 (C, Ph), $129.89(\mathrm{CH}, \mathrm{Ph}), 128.52(\mathrm{CH}, \mathrm{Ph}), 127.69(\mathrm{CH}$, $\mathrm{Ph}), 124.03\left(\mathrm{C}, \mathrm{q}, J_{\mathrm{CF}} 289, \mathrm{CF}_{3}\right), 123.93(\mathrm{CH}, \mathrm{C}-2), 85.01$ $\left(\mathrm{C}, \mathrm{JCCF}_{3} 28, \mathrm{CCF}_{3}\right), 73.79(\mathrm{CH}, \mathrm{C}-6), 70.63(\mathrm{CH}, \mathrm{C}-9)$, $55.49\left(\mathrm{CH}, \mathrm{OCH}_{3}\right), 38.62(\mathrm{CH}, \mathrm{C}-1), 20.20\left(\mathrm{CH}_{2}, \mathrm{C}-2\right)$ and $20.17\left(\mathrm{CH}_{2}, \mathrm{C}-3\right)$; MS: $m / z 370\left(M^{+}, 0.2 \%\right), 301$ $\left.\begin{array}{lllll}M-\mathrm{CF}_{3}, & 0.6\end{array}\right), \quad 233 \quad(0.2), \quad 190 \quad(9.8), \quad 189$ $\left[\mathrm{C}_{6} \mathrm{H}_{5} \mathrm{C}\left(\mathrm{CF}_{3}\right)\left(\mathrm{OCH}_{3}\right), 100.0\right], 158(1.4), 137$ (1.4) and 105 (9.5). (Found: $M^{+}, 370.10180$ ). $\mathrm{C}_{18} \mathrm{H}_{17} \mathrm{~F}_{3} \mathrm{O}_{5}$ requires $M, 370.10280$. The hydroxy lactone $(-)-3$ provided the ester $(+)-8$ with spectral characteristics as described previously.

$X$-Ray structure determination. The crystal data and the details of data collection and structure refinement are given in Table 1 . The intensity data were collected at $140 \mathrm{~K}$ using a Delft Instruments FAST TV area detector diffractometer positioned at the window of rotating anode generator with Mo-K $\alpha$ radiation $(\lambda=0.71069 \AA)$, by following previously described procedures. ${ }^{7} 250$ reflections within $\theta=1.87-24.93^{\circ}$ were used for the refinement of the unit cell parameters. The data were corrected for Lorentz and polarization factors, and also for adsorption using the program DIFFABS. ${ }^{8}$

The structure was solved by direct methods (SHELXS) ${ }^{9}$ and refined on $F^{2}$ by full-matrix least-squares (SHELXL-93) ${ }^{10}$ using all unique intensities above background. The non-hydrogen atoms were all anisotropic; the hydrogen were included in idealised positions with $U_{\text {iso }}$ set at $n U_{\text {eq }}$ of the parent carbons ( $n=1.2$ for tertiary and 1.5 for methyl hydrogens). The weighting scheme given in Table 1 was chosen to obtain satisfactory agreement analyses. Final $w R_{2}$ (on $F^{2}$ ) and $R_{1}$ (on $F$ ) values were 0.0695 and 0.0384 , respectively, for 236 parameters and all 2577 data. The corresponding $R$-indices for 2214 data with $I>2 \sigma(I)$ were 0.0673 and 0.0323 , respectively. All calculations were done on a $486 \mathrm{DX} 2 / 66$ personal computer. Sources of scattering factor data are given in Ref. 10.

The anisotropic displacement parameters of the nonhydrogen atoms and hydrogen atom parameters have been deposited at the Cambridge Crystallographic Data Centre. 
Acknowledgements. We thank the British Council for a grant (to X. H.) and the SERC, the DTI and Chiroscience Ltd. for a post-doctoral Fellowship (to H. F. O.) under the aegis of the Biotransformations LINK Programme. Financial support from Novo Nordisk is gratefully acknowledged (to D. R. V.). The SERC is also thanked for support to the crystallography service at Cardiff.

\section{References}

1. Lubineau, A., Auge, J. and Lubin, N. Tetrahedron Lett. 32 (1991) 7529.

2. MacKeith, R. A., McCague, R., Olivo, H. F., Palmer, C. F. and Roberts, S. M. J. Chem. Soc., Perkin Trans. 1 (1993) 313.

3. Grieco, P. A., Henry, K. J., Nunes, J. J. and Matt, J. E. J. Chem. Soc., Chem. Commun. (1992) 368.
4. Casy, G., Gorins, G., McCague, R., Olivo, H. F. and Roberts, S. M. J. Chem. Soc., Chem. Commun. (1994) 1085; McCague, R., Olivo, H. F. and Roberts, S. M. Tetrahedron Lett. 34 (1993) 3785 and references therein.

5. MacKeith, R. A., McCague, R., Olivo, H. F., Roberts, S. M., Taylor, S. J. C. and Xiong, H. Bioorg. Med. Chem. 1994. In press.

6. For some preliminary results see Garofalo, A., Hursthouse, M. B., Olivo, H. F., Roberts, S. M. and Sik, V. J. Chem. Soc., Perkin Trans. 1 (1994) 1311.

7. Darr, J. A., Drake, S. R., Hursthouse, M. B. and Malik, K. M. A. Inorg. Chem. 32 (1993) 5704.

8. Walker, N. P. C. and Stuart, D. Acta Crystallogr., Sect. A 39 (1983) 158; adapted for FAST geometry by Karaulov, A. I., University of Wales, 1991.

9. Sheldrick, G. M. Acta Crystallogr., Sect. A 46 (1990) 467.

10. Sheldrick, G. M. SHELXL-93 Program for Crystal Structure Analysis, University of Gottingen, Germany, 1993.

Received July 25, 1994. 